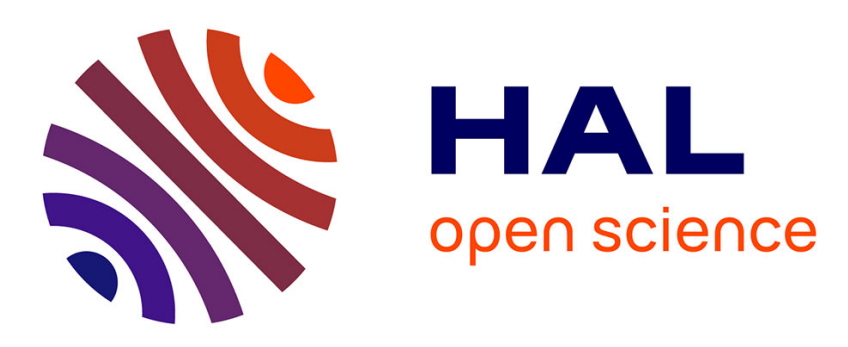

\title{
Flash point of binary mixtures of chlorinated hydrocarbons with toluene and their predictability with existing mixing rule
}

Guillaume Fayet, Benoït Tribouilloy, Patricia Rotureau

\section{- To cite this version:}

Guillaume Fayet, Benoït Tribouilloy, Patricia Rotureau. Flash point of binary mixtures of chlorinated hydrocarbons with toluene and their predictability with existing mixing rule. Process Safety Progress, 2020, pp.art. e12127. 10.1002/prs.12127 . ineris-03318303

\section{HAL Id: ineris-03318303}

https://hal-ineris.archives-ouvertes.fr/ineris-03318303

Submitted on 9 Aug 2021

HAL is a multi-disciplinary open access archive for the deposit and dissemination of scientific research documents, whether they are published or not. The documents may come from teaching and research institutions in France or abroad, or from public or private research centers.
L'archive ouverte pluridisciplinaire $\mathbf{H A L}$, est destinée au dépôt et à la diffusion de documents scientifiques de niveau recherche, publiés ou non, émanant des établissements d'enseignement et de recherche français ou étrangers, des laboratoires publics ou privés. 


\title{
Flash point of binary mixtures of chlorinated hydrocarbons with toluene and their predictability with existing mixing rule
}

\author{
Guillaume Fayet*, Benoit Tribouilloy, Patricia Rotureau \\ Institut National de l'Environnement Industriel et des Risques (Ineris), Parc Technologique \\ Alata, BP2, 60550 Verneuil-en-Halatte, France. \\ *corresponding author: guillaume.fayet@ineris.fr; tel: +33(0)344618126
}

\section{ABSTRACT}

The flash points of binary mixtures of toluene with two chlorinated hydrocarbons (trichloroethylene and dichloromethane) at different concentrations were investigated to evaluate their predictability by existing mixing rules.

Measurements were performed using both open and closed cup flash point testers highlighting complex ignition phenomena. Beyond a certain level of concentration in chlorinated compound, no proper flash is observed, in our test conditions when the flame is presented, but specific behaviors are observed that did not allow the definitive determination of a flash point. The observed phenomena can be attributed to the formation of halogenated radicals, presenting flame-retardant behaviors, by decomposition of the chlorinated vapors at the contact of the flame.

Existing mixing rules are based on the consideration of the vapor-liquid equilibrium defining the amount of flammable vapors above the liquid surface. So, the application of such mixing rule failed predicting this specific phenomenon, more likely related to a specific reactivity problem.

This work also confirms that the sole consideration of the flash point to investigate the flammability of such mixtures may lead to an underestimation of their actual hazard. Additional experimental tests should be conducted to investigate deeper the flammability of such liquid mixtures.

Keywords: flash point; chlorinated solvents; mixtures; UNIFAC 


\section{INTRODUCTION}

Taking advantage of their non-flammability (in particular according to the criteria of hazardous materials regulations), some chlorinated compounds were widely used during the 1950s and 1960s for different applications like degreasing solvents, fertilizers or refrigerants. Unfortunately, such compounds revealed toxic for human and environment. For instance, trichloroethylene, widely used as degreasing solvent [1], presents toxic and carcinogenic concerns [2-4]. For this reason, 1,1,1-trichloroethane has been favored [1]. Unfortunately, this last revealed pointed out as depleting the Ozone layer in the context of the Montreal Protocol [5]. Reporting on the way to address or improve the appraisal of actual flammability hazard of halogenated substances and related mixtures is still of great pertinence according to market development of substitutes (like HFOs...) of banned CFCs and HCFCs which were used in the past as refrigerants, propellants and so forth. Indeed, past research has shown that flammability of such substances and their explored substitutes often revealed underscored by classical measured parameters (mostly flash point) [6].

To reduce their impact to the environment, chlorinated substances can be used into mixtures. But the co-solvent used to form operational chlorinated mixtures can then present or increase the subsequent overall flammable potential. So, the flammability of their mixtures may have to be checked for appropriate fire safety management and relating regulation compliance applicable in given industrial use. If other properties are required for a complete hazard investigation (notably flammability limits) in particular in the case of halogenated mixtures [6], the flash point remains the first experimental data for the classification of flammable liquids in regulations $[7,8]$. The particular flammable behavior of chlorinated mixtures was highlighted yet in the early 1930s [9], but only few flash point data were published in literature. In 1930, Snell investigated mixtures of carbon tetrachloride $\left(\mathrm{CCl}_{4}\right)$ with toluene and acetone [10]. In 1973, Gerstein et al. performed a qualitative investigation of the flammability of kerosene- $\mathrm{CCl}_{4}$ mixtures but without quantitative determination of the flash point [11]. The most complete flash point profiles available in the literature were proposed by Rybicky et al. in 1981 for trichloroethylene, trichloroethane and dichloromethane mixtures with toluene [12]. In this study, both closed and open cup testers were used and, surprisingly, some open cup measurements were found lower than closed cup ones.

To access the flash point of mixtures, predictions can be derived from mixing rules. Among them, the predictive approach of Liaw [13] already presented high accuracy for a large diversity of mixtures with diverse levels of volatility including binary and ternary mixtures $[14,15]$, taking into account possible partial miscibility [16] and aqueous solutions [17, 18]. Within this approach, the flash point of the mixture is based on the accurate estimation of the vapor-liquid equilibrium which depends on the (non-)ideality of the solution. Different models can be used to access activity coefficients: Wilson [19], NRTL [20], UNIQUAC [21]. Within these models, interaction parameters are fitted on vapor-liquid equilibria (VLE) measurements. In the case of the group contribution UNIFAC model [22], activity coefficients are predicted from group 
interaction parameters (themselves fitted on large experimental VLE datasets). Due to the low availability of experimental data of mixtures involving chlorinated compounds, applicability of such mixing rule remains to be checked for mixtures involving halogenated compounds.

In this context, the present study aimed to evaluate the predictive capabilities of the mixing rule when compared to flash point measurements of trichloroethylene and dichloromethane in mixture with toluene at different concentrations.

\section{MATERIALS AND METHODS}

\subsection{Experiments}

Flash point testers are divided into two categories: closed cup and open cup apparatus, according to the types of sample vessel. Moreover, they can follow different modes of temperature controls: by fixed-temperature method or by rising-temperature method [9]. In this study, two different testers were used: a closed cup Setaflash series 3 tester (StanhopeSeta) using a fixed-temperature method and a Cleveland flash point tester (CLA 5, Anton Paar) with an open cup and a rising-temperature method. No correlation can be ensured between results issued from the two used testers.

All such devices anyway give apparatus dependent and when manually operated operatordependent results, likewise for nearly all fire safety relevant parameters. This is due to the fact that flash points, as well as flammability limits, autoignition temperatures etc. are non-intrinsic variables of substances that consequently do not present a true and unique value by contrast to melting points for instance.

For classification purpose, closed cup methods are recommended [9]. Indeed, closed cup methods are conducted in a confined environment. Consequently, the results are less likely influenced by surrounding conditions. Moreover, since the flammable vapors are contained, ignition is observed at lower temperature than in open cup testers, providing more conservative results and ensuring safer practices.

Measurements using the Setaflash tester were conducted according to the ISO 3679 standard test protocol [23] using a fixed-temperature method with 1 min equilibrium time below $100^{\circ} \mathrm{C}$ and $2 \mathrm{~min}$ above $100^{\circ} \mathrm{C}$. The Cleveland tester was used according to the ISO 2592 standard [24] which requires a temperature rate between $5^{\circ} \mathrm{C} / \mathrm{min}$ and $17^{\circ} \mathrm{C} / \mathrm{min}$ until $56^{\circ} \mathrm{C}$ of the expected flash point and, thereafter, between $5^{\circ} \mathrm{C} / \mathrm{min}$ and $6^{\circ} \mathrm{C} / \mathrm{min}$. When no flash was observed, testing was conducted until reaching the boiling point of the liquid mixture. No significant influence of the barometric pressure was found applying the barometric correction proposed by the CEN/TR 15138 guide to flash point testing. The mentioned testers have been selected according to criteria motivations (at least one of each family - closed and open cup) and their relatively wide use at European level. 
It may to be noticed that, considering the specificity of halogenated mixtures, additional tests would have been recommended for classification purpose, like the Lower Explosion Point [25, 26] (also called Lower Temperature Limit of Flammability in ASTM E1232 standard [27]) or the Lower Flammability Limits (as recommended in ASTM E502 standard [28]), following the specific test methods proposed for difficult-to-ignite materials in ASTM E681 [29] and EN 1839 [30] standards. Nevertheless, the mixing rule targets the prediction of flash points. For this reason, the experimental campaign was focused on flash point measurements even if such experiment doesn't necessary meet all existing recommendations for best practice for an appropriate and full assessment of flammability hazards for the studied halogenated mixtures.

A series of binary liquid mixtures of toluene with two chlorinated compounds (trichloroethylene and dichloromethane) were tested at different concentrations. Toluene (with a purity of $100 \% \mathrm{w})$, trichloroethylene $(99.9 \% \mathrm{w})$ and dichloromethane $(100 \% \mathrm{w})$ were purchased at VWR Chemicals Company (Radnor, Pennsylvania, US). The mixtures were prepared in different concentrations from the mass measured using a Setra digital balance (EL410D: sensitivity, $0.001 \mathrm{~g}$; maximum load, $100 \mathrm{~g}$ ). Before testing, each sample was stored for $24 \mathrm{~h}$ in a fridge at $0^{\circ} \mathrm{C}$ in a closed container.

\subsection{Mixing rule}

The full flash point profiles of the two binary liquid mixtures were calculated using the mixing rule of Liaw [13] using Simulis Thermodynamics [31]. As already proposed by Ghmeling and Rasmussen [32], this approach considers the flash point as the temperature at which the vapor phase composition in the air reaches the lower flammability limit (LFL).

Based on this principle, the flash point of a miscible mixture can be estimated by combining the Le Chatelier equation (to estimate the lower flammability limit of the vapor mixture), the Antoine equation (for the dependency of saturated vapor pressures with temperature) and an activity coefficient model (to account for the non-ideality of the liquid mixture). Finally, the flash point is calculated as the temperature satisfying Eq. 1.

$$
\sum_{i} \frac{x_{i} \gamma_{i} P_{i}^{s a t}(T)}{P_{i, F P}^{s a t}}=1
$$

where $x_{i}$ is the mole fraction of flammable substance $i$ in the liquid phase, $\gamma_{i}$ is its activity coefficient in the solution, $P_{i}^{s a t}(T)$ and $P_{i, F P}^{s a t}$ are the saturated vapor pressures of pure flammable compound $i$ at the temperature $T$ and at its flash point and $P$ is room pressure.

In the present case, chlorinated compounds (trichloroethylene and dichloromethane) are nonflammable. So, only toluene contribution to the flash point was considered as done for aqueous mixtures $[17,18]$.

Activity coefficients were evaluated using the UNIFAC model. The Universal Functional Group Activity Coefficient (UNIFAC) model [22] is a group contribution approach that uses group 
interaction parameters to predict the activity coefficient. In this study, the modified Dortmund 93 [33] set of parameters was used, as implemented into Simulis Thermodynamics [31]. This method revealed already powerful for the calculation of activity coefficients to predict the flash point of different kinds of mixtures (organic [32, 34, 35], phosphatic [36], aqueous [17]). Saturated vapor pressures were calculated according to the extended Antoine equations and associated parameters as provided by Simulis Thermodynamics (see Supporting Information).

\section{RESULTS AND DISCUSSION}

Two binary liquid mixtures were investigated: trichloroethylene / toluene and dichloromethane / toluene. In both cases, flash points at different concentrations in chlorinated compound were measured using both the closed cup and open cup protocols.

The flash point of pure toluene was measured at $4.5^{\circ} \mathrm{C}$ and $12.0^{\circ} \mathrm{C}$ with the closed and open cup respectively, which is in agreement with existing available data (presented in Table 1). These two values were used in the prediction method to access the flash point profiles of the two mixtures according to both experimental conditions, even if the predictive approach is dedicated to closed cup flash points. Indeed, in open cup tester, flash point is measured farther from equilibrium conditions. So, larger errors may be expected, predictions underestimating experimental data.

The flash point profile of the trichloroethylene / toluene mixture determined by both experimental and computational approaches is provided in Figure 1. At first, it has to be noticed that the flash points obtained with the Cleveland open cup tester are significantly higher than those obtained with the Setaflash closed cup method. This observation is in contradiction with Rybicky's results [12] but more in agreement with common experimental knowledge [9].

As shown in Table 2, no proper flash point was measured at high concentrations of trichloroethylene (from 63\%). At these concentrations, no flash was observed when the flame was presented above the liquid surface. According to the CEN/TR-15138 standard [37], the flammability criteria is the observation of ignition of the vapors and propagation of the flame over the entire liquid surface. In the open cup apparatus, the flame was enlarged during the time it was presented above the liquid surface but the flame did not cover the whole liquid surface. In the closed cup tester, a blue halo appeared near the flame, out of the cup, but no flash was observed covering the liquid surface inside the cup. In both cases, this phenomenon was not maintained once the flame moved away. As already highlighted by Babrauskas [9], such observations don't allow the determination of a definitive flash point. Nevertheless, it doesn't prevent against possible flammability issues in industrial conditions.

Subjacent phenomenon can be attributed to the flame-retardant behavior of halogenated decomposition products of trichloroethylene [38]. The retarding effect of halogenated compounds is well known and has been notably demonstrated by Tewarson et al. by studying Halion $1301\left(\mathrm{CBrF}_{3}\right)$ as flame extinction solution [39]. In this study, Tewarson observed an 
instability of the flame in presence of Halion 1301 until extinction above a certain concentration, e.g. 5.5\% in PMMA. Lask and Wagner also demonstrated the inhibitor efficiency of different halogenated substances (including $\mathrm{CCl}_{4}$ and $\mathrm{CHCl}_{3}$ ) to decrease the flame velocity of stoichiometric hexane-air mixture [40]. Moreover, chlorinated decomposition products $(\mathrm{HCl}$ and chlorinated benzenes) have been already identified as flame-retardant species in the past in the combustion of chlorinated polymers [41, 42].

Trichloroethylene decomposition reaction was proposed [43] to be the following:

$$
\mathrm{C}_{2} \mathrm{HCl}_{3} \rightarrow \mathrm{C}_{2} \mathrm{HCl}_{2}^{\bullet}+\mathrm{Cl}^{\bullet}
$$

Pyrolytic conditions even favor successive hydrodechlorinations to access non-chlorinated products as demonstrated by Wong et al. by studying the decomposition of trichloroethylene in excess of hydrogen [38]. Besides, similar mechanism was proposed for the decomposition of chloroform [44].

During the flash point testing, the flame is moved over the liquid surface. At the test temperature for which these phenomena are observed (from about $25^{\circ} \mathrm{C}$ ), this temperature is not high enough to form spontaneously chloride radicals from trichloroethylene in the liquid phase or in the vapor phase especially within the time of the test. Nevertheless, the flame can decompose trichloroethylene vapor in the gas phase. This could explain why the flame is enlarged over the whole liquid surface in the open cup tester and why it forms a blue halo in the closed cup tester (due to the accumulation of the halogenated gaseous residues inside the cup).

The anomaly observed on the flash points in open cup, an ignition out of the vessel, was also in line with the anomaly described by Gerstein as caused by the mixing with air [11]. As indicated by Gerstein, such ignition occurs in vapor-air conditions that differs from the expectations of standard procedures and may not alter the validity of the test but it exhibits the fact that some liquids presenting no flash point can explode or burn in certain conditions.

It has to be noticed that, just before the limit at which no flash point can be measured (between $50 \%$ and $63 \%$ in trichloroethylene), an important increase of the flash point is observed in open cup measurements. No such increase is observed for the closed cup measurements. This could be due to the air supply in the open cup measurements and reinforce the relevance of closed cup testers compared to open cup measurements that could lead to underestimate fire safety issues in risk assessment studies.

The results obtained for the dichloromethane/toluene mixtures (in Figure 2) confirmed the observations made for the trichloroethylene/toluene mixture. Indeed, the close cup apparatus revealed again more conservative than the open cup one.

For this mixture, the limit from which no flash point was measured appeared at lower concentrations (from $33 \%$ of dichloromethane) due to the higher volatility of dichloromethane 
(with a boiling point $\mathrm{T}_{\mathrm{b}}$ of $37.9^{\circ} \mathrm{C}$ [45]) compared to trichloroethylene (with $\mathrm{T}_{b}=87.2^{\circ} \mathrm{C}$ [46]) that favored a larger amount of chlorinated compound in the gas phase and, as a consequence, a larger amount of flame-retardant chlorinated radicals formed at the contact of the flame.

The predictive mixing rule is only based on the consideration of a vapor-liquid equilibrium. So, it didn't take into account possible specific reactivity effects, like the formation of flame retardant products in the decomposition of chlorinated compounds. As a consequence, it is not able to predict the specific ignition phenomena observed for the two investigated toluenechlorinated compound mixtures (enhanced flame, in open cup apparatus, and blue halo in close cup apparatus).

Moreover, an underestimation was observed for open cup flash points. Indeed, in this type of tester, the vapors evaporated from the liquid are not contained into the sample vessel whereas the mixing rule relies on a vapor-liquid equilibrium hypothesis. Besides, the mixing rule is neither able to identify the increase of open cup flash points observed in the concentrations near the threshold from which no proper flash point can be measured.

So, even if the mixing rule gives a conservative estimation of the flash point (even when a flash is no more experimentally observed), the observed limitations don't allow to validate its use for halogenated mixtures in a regulatory purpose, as indicated in the Globally Harmonised System of Classification and Labelling of Chemicals (GHS) [7].

More widely, flash point may even be considered with great care when assessing fire hazards of chlorinated mixtures. Indeed, due to the difference in volatility between trichloroethylene and toluene, flash point measurements can underestimate the flammable ability of liquid in some scenarios. Indeed, with a boiling point of $87.2^{\circ} \mathrm{C}$ [46] and $37.9^{\circ} \mathrm{C}$ [47], respectively, trichloroethylene and dichloromethane are more volatile than toluene $\left(110.6^{\circ} \mathrm{C}[46]\right)$. So, in open air storage conditions, chlorinated compounds can evaporate before toluene. The liquid solution will decrease in chlorinated concentration with time and can become flammable due to the loss of the halogenated fraction within the liquid even if the original solution was evidenced to present high or even no flash point.

Moreover, if the observed ignition phenomenon doesn't fit with the standard recommendations for the determination of a flash point, it doesn't prevent against possible fire ignition in industrial conditions of storage and handling, in particular where scenarios may involve ignition sources associated with higher energy levels than those involved in standardized flash points standard procedures that might overcome the fire retardancy effect bound to the chlorine element in the concerned mixtures. Besides, if the only flash point of the substance is required for the classification of flammable liquids in the GHS [7], further characterization of the flammability of the mixture may be recommended for risk assessment purpose. For instance, the US Department of Transport regulations (49 CFR 173.120 [48]) specifies that, for the case of mixtures of compounds presenting different volatilities and flash points, if the flash point of the mixture is higher than $-7^{\circ} \mathrm{C}$, a second test shall be done on a 
partially evaporated substance. For such mixtures, with large difference in volatilities between its components, the predictive mixing rule could also represent a powerful complementary tool to experimental measures by allowing the extrapolation of the full flash point profile of the mixture over the whole range of concentrations. Then, it can allow to evidence in which range of concentrations the mixture can become flammable.

Unfortunately, in the present study, available mixing rules are not able to take into account the flame-retardant effect of the chlorinated radicals produced by the decomposition of chlorinated hydrocarbons at the contact of the flame in the vapor phase. More generally, in the case of halogenated liquids, additional tests should advantageously be conducted. Lower Explosion Points $[26,27]$ would notably allow information at the liquid-vapor equilibrium in larger volumes, notably to investigate the flammability limits of the vapor mixture following the specifications of the EN-1839 [30] and ASTM E681-09 standards [29]. It must be noticed that both standards recommend specific test conditions for halogenated substances. The flammability limits may be notably determined under $50 \%$ relative humidity air conditions. Indeed, this humidity can influence flammability limits by catching the halogenated radicals $X^{\bullet}$ (that can hinder ignition) to form the corresponding $\mathrm{HX}$ acids.

\section{CONCUSION}

The flash point profiles of two toluene / chlorinated hydrocarbon mixtures were investigated with both purposes of screening potential measurement issues of flash point and a first order evaluation of the capability of existing models (mixing rule) to predict the flammability of such halogenated mixtures. Measurements, performed using both closed cup and open cup testers, demonstrated the complex behaviors of such mixtures with particular ignition phenomena that can lead to underestimate the flammability hazard of such mixtures.

The decomposition of chlorinated hydrocarbons forms chlorinated radicals that present flameretardant behaviors. These radicals that can hinder the proper flash ignition above the liquid surface and favor specific phenomena at the contact of the flame (enlargement of the flame without covering of the entire liquid surface in open cup tester and appearance of a blue halo in closed cup one). Such reactivity phenomenon is not taken into consideration in existing mixing rules to predict the flash point of mixtures from knowledge of the one of pure compounds.

More generally, the flash point may not be considered as a unique indicator of flammability hazards for chlorinated liquids since the absence of flash during the test does not prevent definitively from an ignition in industrial situations (notably in open air conditions if the halogenated fraction is more volatile than the flammable one). These conclusions could be also relevant for other kinds of mixtures, notably for those involving other types of halogenated compounds. This work encourages to enlarge the measure of flash point profiles of such mixtures since existing data in open literature are up to now limited to a relatively small diversity of mixtures. Two routes in our mind would deserve to be explored to further 
dig the issues investigated in our paper: one would prioritize the use of other flammability testers considering more specific recommendations covering the flammability assessment of chlorinated substances emitted $[6,49]$ at European side $[26,37]$, and at USA level [27] in terms of trying to consider variation of apparatus dependency of measured results bias as a function of concentration of the chlorinated substance in the mixture: possibly by increasing the ignition energy level some way; on the other hand, likely more difficult however, refining flash point prediction models by the incorporation of physical laws reflecting the fire retardancy of relating radicals quenching the combustion process involved in its early stage.

\section{Acknowledgments}

The authors thank J. Engler, D. Carson and G. Marlair for fruitful discussions.

\section{REFERENCES}

[1] R.E. Doherty, A History of the Production and Use of Carbon Tetrachloride, Tetrachloroethylene, Trichloroethylene and 1,1,1-Trichloroethane in the United States: Part 2--Trichloroethylene and 1,1,1Trichloroethane, Environ. Forensics 1 (2000), 83-93.

[2] "Trichloroethylene, tertrachloroethylene, and some other chlorinated agents", IARC monographs on the evaluation of carcinogenic risks to humans, International Agency of Research on Cancer (IARC), 2014.

[3] "Carcinogenesis bioassy of trichloroethylene", Carcinogenesis Technical Report Series, National Cancer Institute, 1976.

[4] "Trichloroéthylène - Fiche de données toxicologiques et environnementales des substances chimiques", INERIS - Fiche de données toxicologiques et environnementales des substances chimiques, 2017.

[5] "Handbook for the Montreal Protocol Substances that Deplete the Ozone Layer", United Nations Envionment Programme, 2012.

[6] L.G. Britton, K.L. Cashdollar, W. Fenlon, D. Frurip, J. Going, B.K. Harrison, J. Niemeier, E.A. Uralh, The Role of ASTM E27 Methods in Hazard Assessment Part II: Flammability and Ignitability, Process Saf. Prog. 24 (2005), 12.

[7] Globally harmonized system of classification and labelling of chemicals (GHS), Seventh revised edition, ST/SG/AC.A10/30/Rev.7, United Nations, 2017.

[8] Regulation (EC) $N^{\circ} 1272 / 2008$ of the European Parliament and of the Council of 16 December 2008 on classification, labelling and packaging of substances and mixtures, amending and repealing Directives 67/548/EEC and 1999/45/EC, and amending Regulation (EC) N 1907/2006.

[9] V. Babrauskas, Ignition Handbook, Fire Sciences Publishers, 2003.

[10] F.D. Snell, Flash Points of Mixed Solvents, Ind. Eng. Chem. 22 (1930), 893-896.

[11] M. Gerstein, W.B. Stine, Anomalies in Flash Points of Liquid Mixtures, Ind. Eng. Chem. Prod. Res. Dev. 12 (1973), 253-255.

[12] J. Rybicky, J.R. Stevens, Deceptive Closed Cup Flash Points of Solvent Mixtures Containing Chlorinated Solvents, J. Coatings Tech. 53 (1981), 40-42.

[13] H.-J. Liaw, Y.-Y. Chiu, A general model for predicting the flash point of miscible mixtures, J. Hazard. Mater. 137 (2006), 38-46.

[14] H.-J. Liaw, V. Gerbaud, Y.-H. Li, Prediction of miscible mixtures flash-point from UNIFAC group contribution methods, Fluid Phase Equil. 300 (2011), 70-82.

[15] H.-J. Liaw, C.-L. Tang, J.-S. Lai, A model for predicting the flash point of ternary flammable solutions of liquid, Combust. Flame 138 (2004), 308-319.

[16] H.-J. Liaw, T.-P. Tsai, Flash-point estimation for binary partially miscible mixtures of flammable solvents by UNIFAC group contribution methods, Fluid Phase Equil. 375 (2014), 275-285. 
[17] H.-J. Liaw, T.-P. Tsai, Flash points of partially miscible aqueous-organic mixtures predicted by UNIFAC group contribution methods, Fluid Phase Equil. 345 (2013), 45-59.

[18] H.-J. Liaw, C.-T. Chen, V. Gerbaud, Flash-point prediction for binary partially miscible aqueousorganic mixtures, Chem. Eng. Sci. 63 (2008), 4543-4554.

[19] G.M. Wilson, Vapor-Liquid Equilibrium. XI. A New Expression for the Excess Free Energy of Mixing, J. Am. Chem. Soc. 86 (1964), 127-130.

[20] H. Renon, J.M. Prausnitz, Local compositions in thermodynamic excess functions for liquid mixtures, AlChE J. 14 (1968), 135-144.

[21] D.S. Abrams, J.M. Prausnitz, Statistical thermodynamics of liquid mixtures: A new expression for the excess Gibbs energy of partly or completely miscible systems, AIChE J. 21 (1975), 116-128.

[22] A. Fredenslund, R.L. Jones, J.M. Prausnitz, Group-contribution estimation of activity coefficients in nonideal liquid mixtures, AIChE J. 21 (1975), 1086-1099.

[23] ISO 3679 - Determination of flash point - Rapid equilibrium closed cup method, 2015.

[24] ISO 2592 - Petroleum and related products - Determination of flash and fire points - Cleveland open cup method, 2017.

[25] E. Brandes, M. Mitu, D. Pawel, The lower explosion point-A good measure for explosion prevention: Experiment and calculation for pure compounds and some mixtures, J. Loss Prevent. Proc. Ind. 20 (2007), 536-540.

[26] EN 15794 - Determination of explosion points of flammable liquids 2009.

[27] ASTM E1232 - Standard Test Method for Temperature Limit of Flammability of Chemicals, 2019.

[28] ASTM E502 - Standard Test Method for Selection and Use of ASTM Standards for the

Determination of Flash Point of Chemicals by Closed Cup Methods, 2013.

[29] ASTM E681-09 - Standard Test Method for Concentration Limits of Flammability of Chemicals (Vapors and Gases), 2015.

[30] EN-1839 - Determination of the explosion limits and the limiting oxygen concentration(LOC) for flammable gases and vapours, 2017.

[31] Simulis Thermodynamics, 2004-2014, ProSim, http://www.prosim.net/.

[32] J. Gmehling, P. Rasmussen, Flash points of flammable liquid mixtures using UNIFAC, Ind. Eng. Chem. Fund. 21 (1982), 186-188.

[33] J. Gmehling, J. Li, M. Schiller, A modified UNIFAC model. 2. Present parameter matrix and results for different thermodynamic properties, Ind. Eng. Chem. Res. 32 (1993), 178-193.

[34] T. Gaudin, P. Rotureau, G. Fayet, Combining mixing rules with QSPR models for pure chemicals to predict the flash points of binary organic liquid mixtures, Fire Saf. J. 74 (2014), 61-70.

[35] H.-J. Liaw, H.-Y. Chen, Study of Two Different Types of Minimum Flash-Point Behavior for Ternary Mixtures, Ind. Eng. Chem. Res. 52 (2013), 7579-7585.

[36] S. Balasubramonian, R.K. Srivastav, S. Kumar, D. Sivakumar, M. Sampath, U. Kamachi Mudali, R. Natarajan, Flash point prediction for the binary mixture of phosphatic solvents and $\mathrm{n}$-dodecane from UNIFAC group contribution model, J. Loss Prevent. Proc. Ind. 33 (2015), 183-187.

[37] CEN/TR 15138 - Petroleum products and other liquids Guide to flash point testing, 2005.

[38] Y.-S. Won, Thermal decomposition of trichloroethylene under a reducing atmosphere of hydrogen, Korean J. Chem. Eng. 26 (2009), 36-41.

[39] A. Tewarson, M.M. Khan, Extinguishment of Diffusion Flames of Polymeric Materials by Halon 1301, J. Fire Sci. 11 (1993), 407-420.

[40] G. Lask, H.G. Wagner, Influence of additives on the velocity of laminar flames, Sympos.

(International) Combust. 8 (1961), 432-438.

[41] A. Tewarson, F. Chu, F.H. Jiang, Combustion Of Halogenated Polymers, Fire Saf. Sci. 4 (1994), 563574.

[42] R.J. Pasek, D.P.Y. Chang, A.D. Jones, Investigation of Thermal Decomposition of Chlorinated Polymers, Hazard. Waste Hazard. Mater. 13 (1996), 23-38.

[43] T.J. Hardwick, Kinetics of the reaction of cyclopentane with trichloroethylene, Int J Chem Kin 1 (1969), 325-337. 
[44] G.P. Semeluk, R.B. Bernstein, The Thermal Decomposition of Chloroform. I. Products, J. Am. Chem. Soc. 76 (1954), 3793-3796.

[45] M.J. O'Neil, The Merck Index - An Encyclopedia of Chemicals, Drugs, and Biologicals, Royal Society of Chemistry, Cambridge, UK, 2013.

[46] W.M. Haynes, CRC Handbook of Chemistry and Physics, 95th ed., 2015.

[47] M.J. O'Neil, The Merck Index - An Encyclopedia of Chemicals, Drugs, and Biologicals., Royal Society of Chemistry, Cambridge (UK), 2013.

[48] Title 49 - Transportation, Code of Federal Regulation, 49 CFR, Chapter I, section 173.120, US Department of Transport (DOT), 2011.

[49] L.G. Britton, Survey of Fire Hazard Classification Systems for Liquids, Process Saf. Prog. 18 (1999), 225-234.

[50] R.M. Butler, G.M. Cooke, G.G. Lukk, B.G. Jameson, Prediction of Flash Points of Middle Distillates, Ind. Eng. Chem. 48 (1956), 808-812.

[51] International Chemical Safety Cards (ICSC) - Toluene, http://www.inchem.org/documents/icsc/icsc/eics0078.htm.

[52] Material Safety Data Sheet - Toluene MSDS - ScienceLab.com, http://www.sciencelab.com/msds.php?msdsld=9927301. 
Table 1 - Comparison of experimental flash points of toluene with literature data

\begin{tabular}{lll}
\hline Type of apparatus & $\mathrm{FP}\left({ }^{\circ} \mathrm{C}\right)$ & Source \\
\hline Closed cup & 4.5 & this study \\
& 4.4 & {$[50]$} \\
& 4 & {$[51]$} \\
& 4.4 & {$[52]$} \\
\hline Open cup & 12 & this study \\
& 16 & {$[52]$} \\
\hline
\end{tabular}


Table 2 - Experimental flash points of toluene with halogenated compounds

\begin{tabular}{|c|c|c|c|}
\hline \multicolumn{2}{|l|}{ Composition } & \multicolumn{2}{|c|}{$\mathrm{FP}\left({ }^{\circ} \mathrm{C}\right)$} \\
\hline Chlorinated compound & $\%$ vol & Closed cup & Open cup \\
\hline \multirow[t]{15}{*}{ 1,1,1-Trichloroethylene } & 0 & 4.5 & 12.0 \\
\hline & 6 & 7.0 & 16.0 \\
\hline & 10 & 8.5 & 14.0 \\
\hline & 13 & 9.0 & 18.4 \\
\hline & 20 & 10.5 & 20.0 \\
\hline & 23 & 11.5 & 20.1 \\
\hline & 28 & 12.0 & 24.0 \\
\hline & 33 & 13.5 & 22.0 \\
\hline & 37 & 14.5 & 28.0 \\
\hline & 42 & 16.0 & 30.0 \\
\hline & 47 & 17.0 & 28.0 \\
\hline & 52 & 19.0 & 42.0 \\
\hline & 58 & 21.0 & 62.0 \\
\hline & 63 & / & / \\
\hline & 84 & / & / \\
\hline \multirow[t]{7}{*}{ Dichloromethane } & 0 & 4.5 & 12.0 \\
\hline & 7 & $8 / 8$ & $18 / 20$ \\
\hline & 14 & 9.0 & 22.0 \\
\hline & 16 & 9 / 9.5 & $22 / 22$ \\
\hline & 24 & 10 & 33 \\
\hline & 33 & / & / \\
\hline & 35 & / & / \\
\hline
\end{tabular}


Figure 1 - Flash point measurements and predictions of trichloroethylene / toluene mixtures using modified Dortmund 93 UNIFAC model

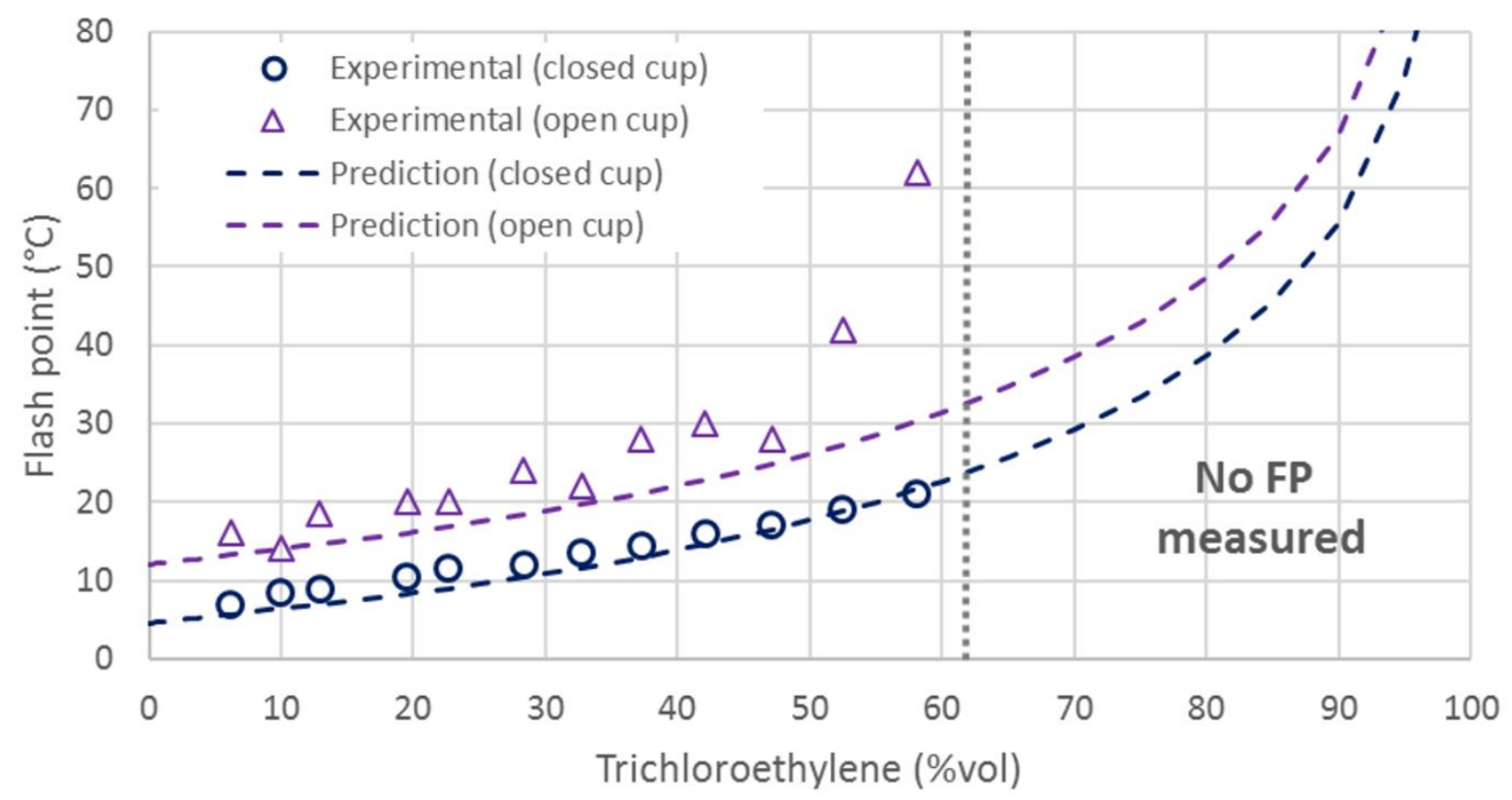


Figure 2 - Flash point measurements and predictions of dichloromethane / toluene mixtures using modified Dortmund 93 UNIFAC model

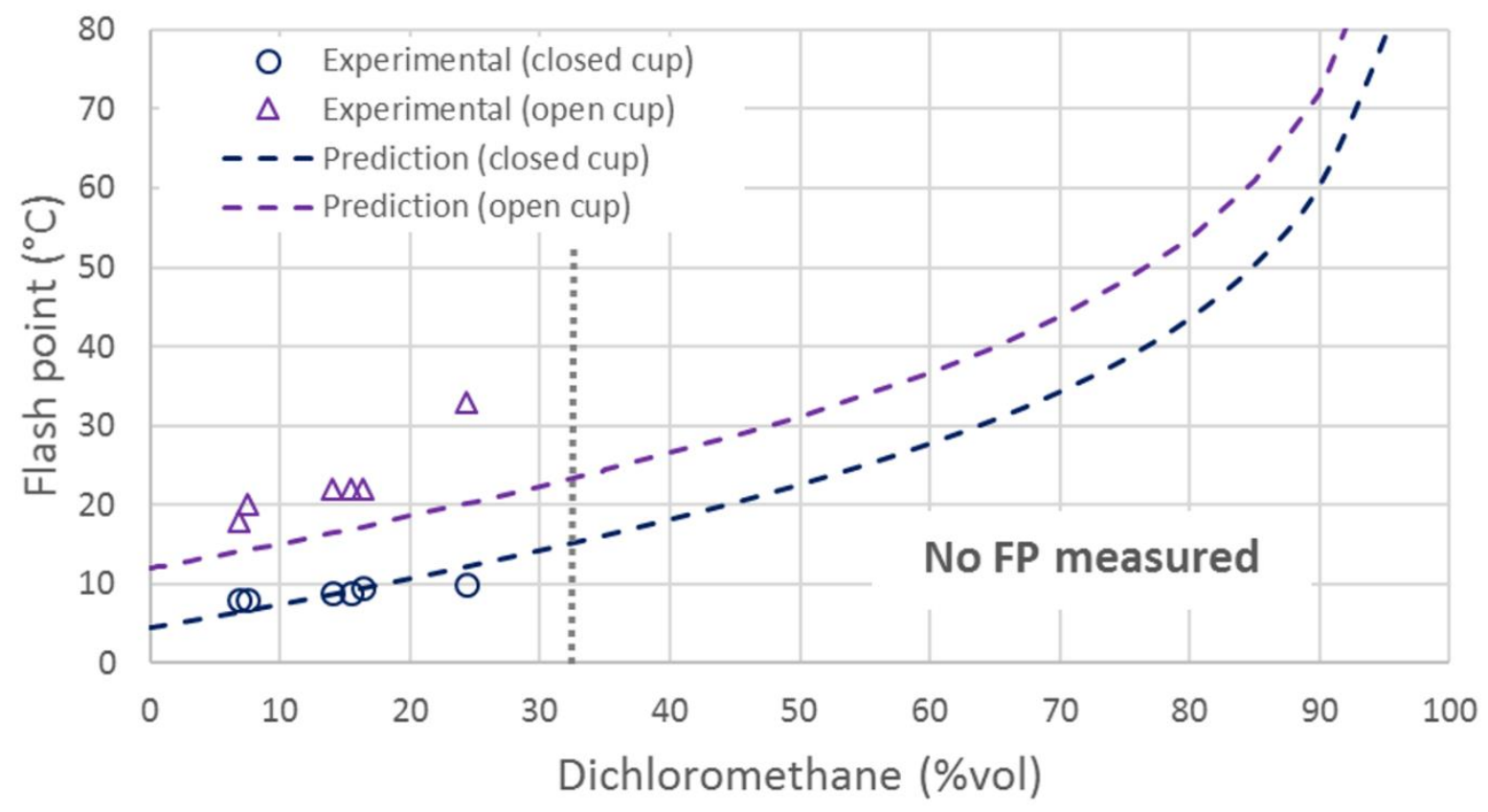

